\title{
LAS ELECCIONES Y EL PARLAMENTO EN EL LIBERALISMO PREDEMOCRÁTICO
}

\section{ELECTIONS AND PARLIAMENT BEFORE DEMOCRACY}

\author{
Roberto Villa García \\ Universidad Rey Juan Carlos
}

Recensión de / Review of: Javier Moreno Luzón y Pedro Tavares de Almeida (Eds.), De las urnas al hemiciclo. Elecciones y parlamentarismo en la Peninsula Ibérica (18751926), Fundación Práxedes Mateo-Sagasta y Marcial Pons Historia, Madrid, 2015, 403 págs.

Palabras clave: Historia Política, Elecciones, Parlamento, Liberalismo, España, Portugal.

Key words: Political History, Elections, Parliament, Liberalism, Spain, Portugal.

El declive de la historia política española, al menos la centrada en el periodo del liberalismo constitucional, ha sido perceptible en los últimos años. La publicación en 2001 de la obra colectiva dirigida por José Varela Ortega, El poder de la influencia. Geografía del caciquismo en España (1875-1923), fue quizás el último gran hito. En ese volumen se ofrecía un análisis regionalizado de las elites politicas del periodo, su perfil profesional y sus redes de sociabilidad. Además, se trataba en profundidad la organización de los partidos de notables y la articulación de un fenómeno tan proteico como el caciquismo. Y, por supuesto, se estudiaba el impacto en el comportamiento electoral tanto de la legislación -sobre todo, la división del territorio y la fórmula de conversión de votos a escaños- como de la práctica del sufragio -ese complejo mecanismo de negociación conocido como "encasillado", o las formas de fraude y corrupción-. A este análisis se le acompañó de una larga conclusión sintetizadora, en la que se manifestaban las diferencias percibidas de la comparación de esos contextos regionales y, más aún, los rasgos comunes que caracterizaron a la vida política española de la Restauración. Como recapitulación de todo lo hecho en los años ochenta y noventa, el avance de conocimiento que supuso esta obra respecto a los estudios realizados durante la Transición fue de tal magnitud que su publicación pareció agotar las líneas de investigación. Un efecto que no era el pretendido por los autores, pues el volumen constituía en realidad un renovado estado de la cuestión, en el que los grandes avances realizados en las postrimerias del siglo XX se entreveraban con abundantes cuestiones abiertas, tareas pendientes y, por tanto, nuevos desafios susceptibles de haber incentivado una mayor continuidad en las investigaciones sobre el periodo.

En un contexto historiográfico en el que, desde 2001, la historia política del XIX y del primer cuarto del XX ya no es una prioridad, y en el que sólo el esfuerzo aislado de unos pocos historiadores la mantiene viva, es estimulante la publicación 
en español de la obra colectiva coordinada por Javier Moreno Luzón (Universidad Complutense de Madrid) y Pedro Tavares de Almeida (Universidade Nova de Lisboa), Das urnas ao hemiciclo, que vio la luz en Portugal hace dos años. Quizás una de esas vías idóneas para ser exploradas con asiduidad es la de la comparación de la Monarquía liberal española con otros sistemas representativos coetáneos. El uso del método comparativo tiene la virtud no sólo de continuar desdibujando las viejas tesis sobre la excepcionalidad española dentro de Europa, aún en boga, sino también de señalar y comprender mejor los aspectos específicos que caracterizaron la trayectoria histórica de la Restauración. En este sentido, la comparación comenzó a dar sus frutos en sendas publicaciones que analizaban las experiencias representativas de España e Italia: la que coordinaron Rosa Ana Gutiérrez Lloret, Rafael Zurita Aldeguer y Renato Camurri (Elecciones y cultura politica en España e Italia (1890-1923), Valencia 2003); y nuevamente Rafael Zurita Aldeguer y Renato Camurri (Las elites en Italia y España, 1850-1922, Valencia, 2009). La monografía que reseñamos añade una nueva referencia de comparación, Portugal, que es especialmente valiosa para los historiadores españoles por las afinidades que, a priori, pueden establecerse en la historia contemporánea de ambos países. Pero también porque enriquece notablemente los elementos de juicio que se tienen para evaluar el recorrido del liberalismo constitucional en nuestro país.

El libro se compone de diez capítulos ordenados en tres secciones. La primera engloba los trabajos de Carlos Dardé, Pedro Tavares de Almeida y María Antonia Peña y María Sierra, y es una historia de la legislación y la práctica electorales, y un esbozo prosopográfico de las elites parlamentarias de ambos países. La segunda se compone de los trabajos de Miguel Martorell, Paulo Jorge Fernandes, Javier Moreno Luzón y Paulo Silveira y María Manuel Tavares, y es una historia del funcionamiento interno y del papel político de los parlamentos español y portugués, incluyendo un pormenorizado análisis de la relación institucional de las Cámaras con la Corona y los Ministerios. Incluye, asimismo, un estudio riguroso de la imagen de ambos parlamentos, de las formas de representación coetáneas, tanto literarias como gráficas, de los Cuerpos colegisladores, de los propios parlamentarios, de cómo eran elegidos y cómo ejercian sus tareas legislativas y fiscalizadoras. Por último, la tercera sección del libro presenta, por un lado, el capítulo de Fernando Catroga, que es un análisis del sistema político de la Primera República portuguesa a partir del "contramodelo" que para aquellos republicanos constituyó la Monarquía recién depuesta; y, por otro, sendos capítulos, de Joao B. Serra y Carlos Dardé, sobre las percepciones y evaluaciones del parlamentarismo liberal centradas, básicamente, en las etapas posteriores a su extinción. Específicamente, Dardé concede un espacio relevante no sólo a los actores políticos, sino también a la propia historiografia y a la evolución de los paradigmas interpretativos sobre la Restauración.

Confieren un valor añadido a esta obra no sólo la presencia de reputados especialistas, con una destacada trayectoria investigadora centrada en este periodo, o la gran cantidad de elementos de análisis con que pueden contar los historiadores en esta apretada sintesis. Sino también, y esto es lo que evidencia el excelente empleo del método comparativo y la utilidad de la monografia, la notable cohesión metodológica del grupo. Esto ha permitido que el libro no constituya un simple sumando de trabajos, sino una obra bastante coherente, que permite cotejar las 
mismas variables en los capítulos dedicados a uno y otro país. El lector puede hacerse una idea bastante clara no sólo de la multiplicidad de aspectos compartidos, sino sobre todo de los factores que explican el recorrido histórico singular de ambos paises y las mutuas influencias y retroalimentaciones, que se prolongan más allá de los años objeto de este estudio.

Esta relativa cohesión metodológica facilita la labor de reseña, que puede hacerse por bloques más que por capítulos, con la finalidad de poner en común los resultados del trabajo los autores. Así, respecto del primer bloque, los capítulos de Dardé y Almeida sugieren semejanzas importantes en la legislación electoral de ambos países, más estrechas quizás durante la Monarquía liberal portuguesa, tanto en el diseño de las circunscripciones como en las fórmulas de reparto de escaños. Igualmente, la analogía se refuerza si se observan las características comunes en el comportamiento electoral (apatía ante las urnas, baja concurrencia de candidatos, hegemonía de las maquinarias caciquiles) y en la manifestación frecuente de prácticas que distorsionaban la libre emisión del sufragio con el objetivo de lograr un resultado ya prefijado (el uso torticero del poder público para hacer triunfar la "candidatura ministerial" y un amplio repertorio de usos fraudulentos y corruptos). En este sentido, los autores resaltan que en ambos países era la Corona, y no las elecciones, la clave de la alternancia. Las votaciones sólo constituían una especie de rito de legitimación ulterior de los que ya gobernaban. Sin embargo, parece que la Corona española tuvo menos margen de maniobra que la portuguesa. Y no tanto por el hecho de que sus prerrogativas estuviesen constitucionalmente más limitadas, sino por su mayor sometimiento a ciertas normas consuetudinarias instituidas en el modelo originario de la Restauración, y que definían los criterios para determinar la alternancia y la disolución de las Cortes.

En España y Portugal es claro, por tanto, que las elecciones no repartían el poder. Sólo lo sancionaban y legitimaban. Pero también funcionaron como mecanismo de integración que encauzaba el disenso -o el conflicto abierto si se trataba de fuerzas politicas a extramuros del sistema político, caso de los republicanos o los carlistas- y como instancia de reclutamiento y renovación de las elites politicas. En este último aspecto, la comparación se diluye un tanto por el desequilibrio entre la información acumulada y las posibilidades de análisis. Precisamente por ello, es dificil sacar conclusiones del tratamiento conjunto de los trabajos de Almeida y de Peña y Sierra. Da la impresión de que en Portugal, en línea con otras historiografias europeas, los especialistas poseen una base de datos bastante completa, y de ahí que puedan trazar los perfiles profesionales y la extracción social del conjunto de los parlamentarios del periodo. Por tanto están en condiciones de articular análisis complejos sobre las diversas formas de encuadramiento político y agregación de intereses. Por el contrario, en España parece que el banco de datos disponible se reduce a una región y, parcialmente, a dos más. Y con una muestra tan reducida es dificil llegar a conclusiones que luego puedan ser objeto de comparación. Con lo disponible, Peña y Sierra han intentado un aproximación provisional al perfil de la elite parlamentaria española pero, como reconocen, "de ninguna forma estamos en condiciones de proporcionar un estudio rigurosamente prosopográfico" (p. 45). 
Pese a que se poseen más datos, algo de esto también se trasluce en los capitulos de Dardé y Almeida, dentro de este primer bloque. De su lectura la impresión que se saca es que, más que diferencias entre ambos regimenes representativos, que las hay, parece que sobre todo existen ritmos diferentes en cuanto a la acumulación de evidencias empíricas y, por tanto, en las posibilidades de articular análisis complejos sobre aquellas realidades políticas. En efecto, ambos capitulos son comparables en la descripción de la naturaleza clientelar de la movilización política y en el análisis de los aspectos formales de la elección, esto es, la legislación electoral, los sistemas electorales que consagraba y su fundamentación política. Pero las diferencias son notables cuando los autores pasan a analizar la práctica electoral. Almeida es, en sus explicaciones, inductivista. Puede ofrecer datos concluyentes sobre la participación electoral, el impacto de la legislación en el comportamiento electoral, el marco de competencia y los niveles de competitividad, la intensidad del intervencionismo gubernamental en las elecciones y su influjo a la hora de procurar el triunfo de unos candidatos sobre otros. Sin embargo, Dardé es más bien deductivista. Analiza la práctica electoral a partir de una explicación general basada en los testimonios de los politicos y publicistas del periodo, y mucho menos de evidencias empíricas. La razón es bien sencilla: de donde no hay, no se puede sacar. Es lo mismo que ocurre con los estudios prosopográficos. Obviando algunos buenos estudios electorales de carácter parcial -ya sea porque estudien una sola elección o varios procesos en un territorio determinado- si se tiene la pretensión de articular un esquema general sobre la práctica electoral en la España de la Restauración, los Diarios de Sesiones y la monografía de Martínez Cuadrado es todo el instrumental que se tiene. $Y$ con eso no se puede alcanzar la profundidad analitica a que se ha llegado en Portugal, como en la mayoría de los países de nuestro entorno.

Y es que, aunque la explicación general que articula Dardé es coherente y rica en matices, el autor reconoce que los estudios parciales que se han realizado matizan, por no decir que echan abajo, algunas de las preconcepciones construidas a partir de los testimonios de aquellos políticos y publicistas. Un buen ejemplo es la afirmación de que los resultados electorales eran un mero epifenómeno de la omnipotencia gubernativa, con su corolario de fraude. El excelente estudio de Dardé sobre las elecciones de 1886 o el de Gutiérrez y Zurita sobre las de 1907 muestran la existencia de coyunturas donde el gobierno ganó las elecciones sin necesidad de emplear a mansalva los resortes administrativos, con un grado de competencia notable y una relativa autenticidad de los resultados en no pocos distritos y circunscripciones. Un caso extremo fue el de las elecciones de 1879, anteriores al turno de partidos y celebradas bajo el gobierno Martínez Campos-Silvela, en el que no hubo encasillado ni se dirigieron las elecciones desde el Ministerio de la Gobernación, ni tampoco hubo un grado significativo de fraude o corrupción. Y el resultado fue, igualmente, el triunfo de los conservadores en el poder. Ciertamente, no todo el fraude era producto del gobierno central pues, aún cuando este se abstuviera, se conoce que los caciques y las autoridades provinciales y locales podian conjurarse para sacar, tirando de trampas, al candidato de su preferencia. Pero con eso pasa lo mismo que con la intervención gubernativa. No basta con 
afirmar que eso ocurriera, sino que hay que verificar su impacto en los resultados, como han hecho los especialistas portugueses.

El expediente, para medir el grado de intervención gubernamental, no se resuelve constatando que los partidos en el gobierno triunfaron en 20 de las 21 elecciones celebradas entre 1876 y 1923. Aparte de que ese dato es discutible, si se tiene en cuenta sobre todo la fragmentación parlamentaria del periodo final de la Restauración, confunde más de lo que aclara. Por poner un ejemplo ilustrativo, en nuestra democracia actual la intervención gubernativa en las elecciones, tal y como estaba articulada en el XIX, ya no existe. Sin embargo, los partidos han seguido ganando muchas más elecciones desde el poder que desde la oposición, sean generales, autonómicas o locales. Más aún, de las primeras seis elecciones generales entre 1977 y 1993, sólo las de 1982 conllevaron el triunfo de un partido de la oposición y en un contexto de bajo nivel de competitividad-que no de competencia-, dadas las proporciones de la victoria del PSOE, aumentadas por la descomposición previa de UCD. Esta comparación diacrónica debería convencernos de que, para comprender la práctica electoral en la Restauración, continuamos necesitando explicaciones con un grado de complejidad y profundidad mayor, que sólo la acumulación de evidencias empíricas nos podría dar.

La comparación es notablemente fructífera en la segunda parte del libro. En efecto, los capitulos de Martorell y Fernandes reflejan unos parlamentos en los que la función legislativa estaba subordinada a la fiscalización del poder ejecutivo quizás esto último con mayor énfasis en España-, y en el que las mayorías parlamentarias no podían imponer su número a unas minorias con mecanismos reglamentarios muy eficaces a la hora de obstruir el programa legislativo de un gobierno. Éste, si quería coronar con éxito su empeño y no encontrarse con oposiciones irreductibles, tenía que pactar no ya el contenido de las leyes sino, previamente y entre bambalinas, el modo en que éstas debían ser discutidas. Además, las evidencias aportadas por los autores destacan cómo las Cámaras tuvieron un papel relevante dentro del sistema politico. En España, con algo más de intensidad que en Portugal, la confianza parlamentaria se constituyó como una condición fundamental para la continuidad del gobierno. Así, la cantidad de ministerios españoles que cayeron en el parlamento lo evidencia de forma palmaria. De ese modo se demuestra que el principio doctrinario de la doble soberanía de la Corona y las Cortes, instituido por la Constitución de 1876, no constituía el diseño subrepticio de una especie de puerta de atrás por la que pudiera colarse el despotismo regio. De hecho, como Martorell destaca para España, el papel constitucional de la Corona en la Restauración apenas difería del modelo del Sexenio. En Portugal lo que se observa, por el contrario, es una mayor supeditación de las Cámaras a la iniciativa de la Corona y del poder ejecutivo, observable por la mayor correlación entre las crisis de gobierno extraparlamentarias y la subsiguiente convocatoria de elecciones. Una experiencia que quizás pueda asemejarse a periodos concretos de la Monarquía constitucional española en sus etapas isabelina o amadeísta.

Las posibilidades de comparación entre el capítulo de Moreno Luzón y el de Silveira y Tavares son amplias. Sin duda, no es extraño que dos sistemas politicos 
con déficits tan palmarios en sus mecanismos representativos fueran tan acerbamente criticados. Ambos trabajos muestran como el caciquismo rural fue un tema recurrente en la literatura política del momento, achacándosele la injusticia, el atraso y la pervivencia incluso de residuos feudales y clericales muy Ancien Régime. El cacique era dibujado como un tiranuelo que explotaba la incultura y la indefensión de los aldeanos para satisfacer su codicia económica, su afán de dominio e, incluso, sus impulsos sexuales. La elite parlamentaria, que permitía este estado de cosas porque necesitaba a los caciques, tampoco se libró de la diatriba. Estaba formada por gentes ociosas, desvergonzadas, corruptas, proclives a la perorata parlamentaria como recurso de lucimiento personal, y completamente ajenas a los problemas del país. Explotadores de un pueblo "honrado, famélico y harapiento... sometido y pasivo ante su desgracia" (p. 218), al que ni siquiera representaban, pues las elecciones eran sistemáticamente falseadas.

Que una denuncia del sistema político tan descarnada y reiterativa pudiera tener lugar desde luego acreditaba un clima efectivo de libertad de expresión. Aspecto que no debería ser obviado, sobre todo por la tendencia que existe en algunos estudios de equiparar estos regímenes subcompetitivos, pero libres, con los autoritarios que les sustituyeron durante el siglo XX, tanto en la península ibérica como en la mayor parte del resto de Europa. Dicho esto, el lector especializado podrá apreciar cómo esta literatura no constituía simplemente el reflejo crítico de los desajustes institucionales y de un orden social abusivo. Precisamente este aspecto la equipara un tanto con la crítica que se ejerce hoy contra el funcionamiento de las instituciones democráticas, por lo menos en cuanto a su intensidad y significación política. Y que cuenta no sólo con gran predicamento en las tertulias televisivas o en la prensa, sino con una bibliografía específica. La intensidad de la crítica no es sorprendente. Conviene recordar que en las sociedades libres, la percepción de los defectos del sistema político suele ser más palpable, la corrupción es más visible y, por tanto, la indignación es mayor y puede expresarse con más efectividad. En cuanto al significado político de la crítica, se evidencia en las formas y el fondo. Cuanto menos empírica y matizada y, por tanto, más descarnada y maniquea es, más se adivina como un mecanismo de deslegitimación. Esto deja ver claramente que la denuncia, sobre todo la articulada a partir de 1898, no tenía como fin en sí misma hacer visibles los defectos del liberalismo representativo. Por el contrario, ésta constituía un medio de subvertirlo, creando un ambiente favorable a su sustitución.

No pueda extrañar, por tanto, que la reprobación de la Monarquía liberal tuviera mucho de estereotipada y artificiosa, basada más en el tópico, la recreación simplista y el análisis superficial que en un ejercicio serio de delimitar los defectos del régimen parlamentario. Tampoco sorprende que buena parte de quiénes la ejercieron tuvieran una militancia política en las formaciones politicas que actuaban en los márgenes del sistema. $\mathrm{Y}$, sin embargo, esta literatura tuvo un impacto importante en la opinión pública. No es que fuera, en última instancia, la responsable de que se cerrase el telón parlamentario durante mucho tiempo en España o Portugal. Pero sí contribuyó a que su caída se hiciera entre la indiferencia general. Por cierto, otra demostración de su eficacia es que aquellos estereotipos literarios perduran hoy, y con mucha fuerza, gracias al cine o la televisión, que llevó 
a la pantalla los relatos, entre trágicos y caricaturescos, de Jarrapellejos o Entre Naranjos. Su reflejo se aprecia incluso en producciones que abarcan etapas posteriores, como Los Jinetes del Alba o La Forja de un Rebelde.

En el tercer bloque, el capitulo de Catroga es una aportación importante para entender la institucionalización del sistema político de la Primera República portuguesa, es decir, las pautas a que responde su modelo originario y que explican su intrincada evolución posterior. Su lectura, sin embargo, consolida la percepción que lo dificil que es establecer una comparación entre aquel régimen y el sistema español de la Restauración, más allá del cotejo de la legislación y la práctica electorales. La constitución de una República parlamentaria con un presidente con pocos poderes efectivos, la constante inestabilidad de los gobiernos, el diseño extremadamente partidista de las reglas electorales y el exclusivismo politico, acompañado de niveles notables de violencia politica, sugieren quizás que la comparación rendiría más frutos si la referencia española fuese el Sexenio Revolucionario o la Segunda República, con la que parece compartir no pocas semejanzas.

Sí que ofrecen mayores posibilidades de comparación los dos últimos capítulos. Básicamente, analizan las visiones postreras de la experiencia constitucional en España y Portugal. Sin embargo, existe una gran desigualdad entre los capítulos. E1 de Serra es, básicamente, un análisis del influjo de la Primera República y sus avatares en el modelo originario de la Segunda, ceñido exclusivamente a la percepción de los políticos constituyentes. El de Dardé, por el contrario, es una amplia y excelente reconstrucción de toda la trayectoria histórica de aquellas visiones, no sólo desde los medios políticos sino también desde los académicos. La afinidad más importante entre los dos países es que, al establecerse en ambos regímenes autoritarios de larga duración, se proyectó durante más tiempo la percepción negativa sobre el liberalismo. Algo que no fue, sin embargo, creación intelectiva de estos regímenes, que se limitaron a reelaborar y reactualizar los estereotipos anteriores. Éstos habían estado plenamente vigentes además durante la Primera República portuguesa y la Segunda República española, pues sus elites politicas también pretendieron desmarcarse de las Monarquías constitucionales a las que habian sustituido.

Aquí, la introducción de la Primera República portuguesa dentro del marco de reflexión quizás distorsione un tanto el análisis. Como resalta Serra, fue la experiencia republicana, más que la monárquica, la que se tomó como una especie de "contramodelo" de la democracia portuguesa instituida en los setenta. En España, la percepción algo más positiva de la Monarquía liberal devino no tanto de su funcionamiento, pues la crítica al caciquismo se mantuvo incólume y hasta tuvo cierta influencia en la discusión de los elementos del sistema electoral, como de su modelo originario transaccional, útil para reforzar a quiénes defendian el tránsito pacífico y pactado desde el franquismo a la democracia. Pero es que, además, los defectos de la Restauración se mantuvieron en un segundo plano porque en la Transición española el "contramodelo" fue la Segunda República, con su corolario de Guerra Civil. Y lo fue no sólo para los políticos de UCD o AP sino claramente para los del PSOE y, con matices, también para el PCE o los partidos nacionalistas. De 
ahí que, igual que en Portugal, los debates historiográficos más enconados y politizados se hayan mantenido precisamente entre los especialistas del periodo republicano, mientras que no han afectado, por lo menos con la misma intensidad, al periodo de la Monarquía constitucional.

En definitiva, estamos ante una monografia sugerente, que consolida la vía de los estudios comparados, quizás uno de los desafios más importantes de la historiografía española en los trabajos sobre la vida política del XIX y del XX. En concreto, la referencia portuguesa demuestra ser de especial utilidad para entender nuestra propia historia politica. Y ha sido mérito de los autores saber explotar esa veta logrando lo más dificil: cierta comunidad de intereses y una importante cohesión metodológica. Ojalá la obra reseñada constituya un acicate para nuevos trabajos sobre la que, obviando el periodo actual, continúa siendo la experiencia constitucional más duradera y relevante de la historia española.

Enviado el (Submission date): 19/04/2015

Aceptado el (Acceptance date): 7/05/2015 